\title{
Insulinomas pancreáticos. Experiencia de seis años en la Clínica de Cirugía Hepatopancreatobiliar del Hospital General de México
}

\author{
Pancreatic insulinomas. Six-year experience in the Hepatopancreatobiliary \\ Surgery Clinic of the General Hospital of Mexico \\ Óscar Chapa-Azuela,* Carmen Roca-Vásquez,* Gustavo Alain Flores-Rangel,* \\ Zazil Botello-Hernández,* Rodolfo Fuentes-Reyes*
}

Palabras clave:

Tumores,

neuroendocrinos, páncreas, insulinoma.

Key words:

Tumors,

neuroendocrine, pancreas, insulinoma.

\footnotetext{
* Cirugía

Hepatopancreatobiliar, Hospital General de México.
}

Recibido: 15/10/2016 Aceptado: 27/10/2017

\section{RESUMEN}

Antecedentes: Los insulinomas pancreáticos pertenecen a un grupo heterogéneo de neoplasias conocidas como tumores neuroendocrinos. Su incidencia aproximada es de 1.8 a cinco casos por 1,000,000 de habitantes y conforman entre el uno y $2 \%$ de todas las neoplasias del páncreas. Pueden tomar un comportamiento benigno o maligno, aunque son la estirpe histológica asociada al mejor pronóstico, con una supervivencia hasta del $97 \%$ a cinco años para tumores benignos y de un 30\% para tumores malignos. Material y métodos: Estudio retrospectivo, observacional, descriptivo, longitudinal, en el cual se analizaron los expedientes de todos los pacientes diagnosticados con insulinoma de páncreas en un periodo de seis años, con un seguimiento en promedio de 32 meses. Resultados: Se encontró un total de siete casos, de los cuales el $100 \%$ fueron benignos y funcionales. Todos los pacientes fueron tratados quirúrgicamente, con intención curativa, con enucleación o resección, sin presentarse complicaciones transoperatorias. En el seguimiento, ninguno mostró datos de recidiva clínica, serológica o imagenológica. Conclusiones: Los insulinomas son tumores que se presentan con baja frecuencia; por lo general son funcionales y se asocian a un buen pronóstico.

\section{ABSTRACT}

Background: Pancreatic insulinomas belong to a heterogeneous group of cancers known as neuroendocrine tumors. Their incidence is approximately 1.8 to five cases/ million inhabitants; they represent between one and $2 \%$ of all pancreatic neoplasms. They can take a benign or malignant course, although insulinoma is the histological type associated with the best prognosis, with up to a $97 \%$ survival at five years for benign tumors and 30\% for malignant tumors. Material and methods: Retrospective, observational, descriptive, longitudinal study in which we analyzed the records of all patients diagnosed with pancreatic insulinoma over a period of six years, with an average follow-up of 32 months. Results: A total of seven cases were found, of which $100 \%$ were benign and functional. All patients were treated surgically with curative intent, either with enucleation or resection, without presenting intraoperative complications. No patient showed clinical, serological or imagenologic data of recurrence. Conclusions: Insulinomas are tumors that occur with low frequency, they are usually functional and associated with a good prognosis.

\section{ANTECEDENTES}

L os insulinomas pertenecen a un grupo heterogéneo de neoplasias denominadas tumores neuroendocrinos (TNE), los cuales derivan embriológicamente de la cresta neural y residen en diversos sitios de los sistemas digestivo y broncopulmonar. ${ }^{1}$

Estos tumores sintetizan y secretan un gran número de péptidos y aminas, las cuales pueden manifestarse clínicamente en una amplia variedad de síndromes derivados de una sobreproducción de hormas determinadas por la estirpe celular que conforma al tumor; la hipoglucemia es la característica clásica de los insulinomas. Por otra parte, una gran cantidad de pacientes pueden permanecer asintomáticos (tumores no funcionales) y manifestarse clínicamente hasta que el volumen tumoral aumenta y aparecen datos de compresión. ${ }^{1}$ 
La incidencia aproximada de los insulinomas es de 1.8 a cinco casos/1,000,000 de habitantes y conforman entre el uno y $2 \%$ de todas las neoplasias del páncreas. ${ }^{1-3}$ Aunque el pico de incidencia ocurre entre los 40 y 69 años de edad, un significativo número de pacientes son diagnosticados antes de los 35 años. ${ }^{4,5}$ Son también los tumores neuroendocrinos asociados al mejor pronóstico, con porcentajes de supervivencia reportados hasta de $97 \%$ a cinco años cuando se trata de lesiones benignas (90\% de los insulinomas lo son) y del 30\% cuando se trata de lesiones malignas. ${ }^{1,6}$

\section{MATERIAL Y MÉTODOS}

Estudio retrospectivo, observacional, descriptivo y longitudinal, en el cual se analizaron todos los casos de pacientes diagnosticados con tumores neuroendocrinos del páncreas de tipo insulinoma referidos a la Clínica de Cirugía Hepatopancreatobiliar del Hospital General de México en un periodo de seis años, que comprendió desde marzo de 2009 hasta junio de 2015, con un seguimiento de los casos en un rango de entre 12 y 72 meses, con una media de 32 .

\section{RESULTADOS}

Se encontraron en total siete casos de insulinoma pancreático, todos ellos sometidos a tratamiento quirúrgico con intención curativa.

Características de los pacientes: Se encontró una mayor incidencia en el género femenino, con cinco pacientes, que representaron un $71.5 \%$ del total de la muestra. La edad media fue de 40 años, con una mínima de 18 y una máxima de 74. Tres pacientes $(42.8 \%)$ presentaron comorbilidades al mo- mento del diagnóstico; en todos los casos fue hipertensión arterial sistémica y ninguno de DM2 previa al manejo quirúrgico. Una paciente cursaba con un embarazo de 19.3 semanas de gestación.

Manifestaciones clínicas: El 100\% de los pacientes presentaron tumores funcionales con manifestaciones clínicas derivadas de hiperinsulinismo, lo cual orientó al diagnóstico en todos los casos; no hubo ningún diagnóstico incidental por imagenología. Los siete pacientes presentaron la tríada de Whipple de hipoglucemia; los síntomas asociados fueron, en orden de frecuencia, astenia, somnolencia, diaforesis, convulsiones y palpitaciones (Cuadro I).

Diagnóstico: A todos los pacientes se les realizó un test bioquímico que incluyó niveles séricos de insulina, glucosa y péptido $\mathrm{C}$, para posteriormente calcularse el índice insulina/glucosa (Cuadro II). Se les hizo también una tomografía de abdomen, donde se logró identificar el tumor primario. En el $100 \%$ de los casos se integró el diagnóstico de insulinoma antes del tratamiento quirúrgico. Existió una correlación del $100 \%$ entre el diagnóstico preoperatorio de insulinoma y el sitio anatómico del tumor con el resultado del estudio histopatológico posterior a la resección.

La localización anatómica del tumor fue, en orden de frecuencia: tres casos en la cola del páncreas $(42.8 \%)$, dos en la cabeza (28.5\%), uno en el cuello (14.2\%) y uno en el cuerpo (14.2\%) (Cuadro III).

Tratamiento: Tres casos (42.8\%) fueron tratados mediante enucleación, con tumores ubicados en distintos segmentos del páncreas (uno en cabeza, uno en cuello y uno en cola), tomándose como criterio de selección una localización periférica (distante al conducto de Wirsung); la enucleación fue exitosa en un

Cuadro I. Cuadro clínico.

\begin{tabular}{lcccccc} 
& Astenia & Tríada de Whipple & Somnolencia & Diaforesis & Convulsiones & Palpitaciones \\
\hline Pacientes & 7 & 7 & 6 & 5 & 5 & 1 \\
Proporción & $100 \%$ & $100 \%$ & $85.7 \%$ & $71.5 \%$ & $71.5 \%$ & $14.2 \%$ \\
\hline
\end{tabular}

Tríada de Whipple: signos clínicos de hipoglucemia que remiten tras la administración de glucosa. 
$66.6 \%$ de los casos (dos pacientes), ya que un paciente requirió posterior pancreatectomía distal por enfermedad residual (Cuadro III).

La cirugía de resección se realizó en cinco pacientes; en cuatro de ellos, como primera intención terapéutica, y en uno, posterior a una enucleación con enfermedad residual R1. El tipo de cirugía se determinó con base en la localización anatómica del tumor, de tal forma que se realizaron cuatro pancreatectomías distales y un procedimiento de Whipple. Se llevó a cabo esplenectomía en cuatro pacientes $(57.1 \%)$, siendo el criterio para realizarla la localización del tumor (en cuerpo y cola), con compromiso de los vasos esplénicos.

El abordaje fue laparoscópico en tres casos $(42.8 \%)$; se requirió conversión en uno. No hubo complicaciones transoperatorias. El tiempo quirúrgico promedio fue de 4.7 horas, con un rango de tres a nueve. El sangrado promedio fue de $176 \mathrm{ml}$, con un mínima de 30 y un máximo de 730 (Cuadro III).

El resultado del estudio histopatológico confirmó el diagnóstico de insulinoma en todas las muestras, con márgenes libres en el $100 \%$ (en un caso, inicialmente se reportó R1, por lo que se reintervino en el mismo internamiento;

\begin{tabular}{lcccc}
\multicolumn{5}{c}{ Cuadro II. Laboratorios preoperatorios. } \\
& $\begin{array}{c}\text { Insulina } \\
\mathbf{m c U} / \mathbf{m l}\end{array}$ & $\begin{array}{c}\text { Glucosa } \\
\mathbf{m g} / \mathbf{d l}\end{array}$ & $\begin{array}{c}\text { Insulina/glucosa } \\
\mathbf{m c U} / \mathbf{m l} \text { - } \mathbf{m g} / \mathbf{d l}\end{array}$ & $\begin{array}{c}\text { Péptido C } \\
\mathbf{n g} / \mathbf{m l}\end{array}$ \\
\hline Media & 24.7 & 38 & 0.81 & 5.02 \\
Mínima & 5.7 & 32 & 0.7 & 3.4 \\
Máxima & 41.4 & 50 & 1.63 & 10.3 \\
& & & & 7 \\
Fuera de rango normal & 5 & 7 & $28.5 \%$ & $100 \%$
\end{tabular}

\section{Cuadro III. Cirugías.}

\begin{tabular}{|c|c|c|c|c|c|c|c|c|c|}
\hline & Sitio & Cirugía realizada & $\begin{array}{l}\text { Esplenecto- } \\
\text { mía }\end{array}$ & Tumor & Abordaje & $\begin{array}{l}2^{\mathrm{a}} \\
\text { cirugía }\end{array}$ & $\begin{array}{l}\text { Compli- } \\
\text { caciones }\end{array}$ & Sangrado & Tiempo \\
\hline 1 & Cabeza & Whipple & No & $1.5 \mathrm{~cm}$ & $\mathrm{Ab}$ & No & No & $730 \mathrm{ml}$ & $9 \mathrm{~h}$ \\
\hline 2 & Cola & $\begin{array}{l}\text { 1. Enucleación } \\
\text { laparoscópica } \\
\text { 2. Pancreatectomía } \\
\text { distal } 60 \%\end{array}$ & Sí & $2.0 \mathrm{~cm}$ & $\mathrm{Ab}$ & Sí, por R1 & No & $50 \mathrm{ml}$ & $\begin{array}{l}3 \mathrm{~h} \\
5 \mathrm{~h}\end{array}$ \\
\hline 3 & Cuerpo & $\begin{array}{l}\text { Pancreatectomía } \\
\text { distal }\end{array}$ & Sí & $1.9 \mathrm{~cm}$ & $\mathrm{Ab}$ & No & No & $150 \mathrm{ml}$ & $4 \mathrm{~h}$ \\
\hline 4 & Cabeza & Enucleación & No & $2.6 \mathrm{~cm}$ & $\mathrm{Ab}$ & No & No & $30 \mathrm{ml}$ & $4 \mathrm{~h}$ \\
\hline 5 & Cola & $\begin{array}{l}\text { Pancreatectomía } \\
\text { distal }\end{array}$ & Sí & $2.2 \mathrm{~cm}$ & Conv & No & No & $100 \mathrm{ml}$ & $5 \mathrm{~h}$ \\
\hline 6 & Cuello & Enucleación & No & $1.6 \mathrm{~cm}$ & Lap & No & No & $30 \mathrm{ml}$ & $3 \mathrm{~h}$ \\
\hline 7 & Cola & $\begin{array}{l}\text { Pancreatectomía } \\
\text { distal }\end{array}$ & Sí & $2.6 \mathrm{~cm}$ & Lap & No & No & $145 \mathrm{ml}$ & $5 \mathrm{~h}$ \\
\hline
\end{tabular}

Ab = cirugía abierta; Lap = cirugía laparoscópica; Conv = laparoscópica convertida a abierta; $\mathrm{R} 1$ = enfermedad residual microscópica. 
en la segunda pieza quirúrgica se consiguió R0). No se reportó invasión linfovascular en ningún paciente. Se realizó inmunohistoquímica en cinco casos, con un $\mathrm{Ki}-67<2 \%$ en cuatro de ellos (G1) y Ki-67 de 6\% (G2) en un paciente con tumor $<2 \mathrm{~cm}$, sin invasión linfovascular. Con los datos disponibles, todos los casos se catalogaron como tumores neuroendocrinos bien diferenciados (OMS).

Evolución: Durante el seguimiento intrahospitalario, tres pacientes precisaron observación en el área de terapia intensiva, con una estancia promedio de 4.3 días, con un rango de tres a siete; las causas fueron neumonía (un caso), control metabólico (un caso) y embarazo (un caso). Se presentaron complicaciones postquirúrgicas en un solo paciente $(14.3 \%)$ debido a infección del sitio quirúrgico, la cual fue resuelta satisfactoriamente con manejo conservador. La media de estancia intrahospitalaria fue de 12 días, con un rango de cinco a 35.

El seguimiento a largo plazo se realizó en un periodo de entre 12 y 72 meses, con una media de 32; en cada consulta se interrogó al paciente y eventualmente se solicitaron controles serológicos y radiológicos. No hubo ninguna defunción y ningún paciente ha presentado datos de recurrencia sintomática, serológica o radiológica. La resolución de los síntomas iniciales de hipoglucemia fue del $100 \%$. Dos casos (28.5\%) desarrollaron datos de falla exocrina del páncreas (diabetes mellitus) (Cuadro IV).

\section{DISCUSIÓN}

\section{Clasificación y estadificación}

En relación con la estirpe histológica, los insulinomas son los TNE pancreáticos más comunes: representan alrededor del $70 \%$, mientras que $15 \%$ corresponden a glucagonomas y $10 \%$ a gastrinomas, somatostatinomas $y$, con menor frecuencia, VIPomas. ${ }^{7}$

Las nuevas clasificaciones proporcionan un gran énfasis al grado de diferenciación histológica del fenotipo neoplásico, reconociéndose tres grados: bajo (G1), intermedio (G2) y alto (G3), de tal forma que con base en el grado del tumor se puede establecer la probabilidad de malignidad (Cuadro V). 2,8,9

En el año 2000, la OMS propuso una clasificación basada en el índice mitótico, el

Cuadro IV. Evolución.

\begin{tabular}{lccccccc} 
& \multicolumn{8}{c}{ Cuadro IV. Evolución. } \\
& $\begin{array}{l}\text { Ingreso } \\
\text { a UCI }\end{array}$ & $\begin{array}{c}\text { Defun- } \\
\text { ciones }\end{array}$ & $\begin{array}{c}\text { Compli- } \\
\text { caciones }\end{array}$ & $\begin{array}{c}\text { Reopera- } \\
\text { ciones* }\end{array}$ & $\begin{array}{c}\text { DM postqui- } \\
\text { rúrgica }\end{array}$ & $\begin{array}{c}\text { Corrección de } \\
\text { la hipoglucemia }\end{array}$ & $\begin{array}{c}\text { Recu- } \\
\text { rrencias }\end{array}$ \\
\hline Total & 3 & 0 & 1 & 1 & 2 & 7 & 0 \\
Proporción & $42.8 \%$ & 0 & $14.2 \%$ & $14.2 \%$ & $28.5 \%$ & $100 \%$ & 0 \\
\hline
\end{tabular}

* Por enfermedad residual R1.

Cuadro V. Sistema de estratificación tumoral propuesta por la

Sociedad Europea de Tumores Neuroendocrinos.

\begin{tabular}{lccc} 
& \multicolumn{3}{c}{ Grados } \\
\cline { 2 - 4 } Índices & G1 (grado bajo) & G2 (grado intermedio) & G3 (grado alto) \\
\hline Mitótico & $<2$ mitosis* & $2-20$ mitosis & $>20$ mitosis \\
Ki-67 & $<2 \%$ & $3-20 \%$ & $>20 \%$ \\
\hline
\end{tabular}

* Número de mitosis en 10 campos de alto poder. Ki-67 = índice de proliferación celular. 
tamaño del tumor y la invasión vascular; con base en estas características, se puede predecir el comportamiento del tumor (Cuadro VI):2,8,9

- Tumor neuroendocrino bien diferenciado de comportamiento benigno.

- Tumor neuroendocrino bien diferenciado de comportamiento incierto.

- Carcinoma neuroendocrino bien diferenciado (tumor carcinoide típico).

- Carcinoma neuroendocrino mal diferenciado (tumor carcinoide atípico).

En la actualidad se ha adoptado el sistema TNM para la clasificación de los TNE gastrointestinales por estadios con base en el tamaño e invasividad del tumor, el compromiso linfático y la presencia de metástasis. ${ }^{2}$

\section{Diagnóstico}

La sospecha diagnóstica varía con base en la funcionalidad del tumor; los tumores funcionales se manifiestan con hipoglucemia derivada del hiperinsulinismo endógeno, mientras que los tumores no funcionales se diagnostican, por lo general, de forma incidental mediante estudios de imagen o por compresión biliar y/o al tubo digestivo secundaria al volumen de la lesión.

La insulina sérica, proinsulina y el péptido $C$ deben solicitarse en todos los casos. ${ }^{10}$ Niveles de insulina mayores a $6 \mathrm{mclU} / \mathrm{ml}$, péptido $\mathrm{C}$ mayor a $0.6 \mathrm{ng} / \mathrm{ml}$, proinsulina mayor a 5
mclU/l, glucosa capilar menor a $55 \mathrm{mg} / \mathrm{dl}$ y una relación insulina/glucosa $<0.3$ sugieren fuertemente la presencia de estos tumores. ${ }^{10}$

Una vez sospechado el diagnóstico por serología, se debe buscar la lesión primaria, para lo cual se sugiere realizar en todos los pacientes una tomografía axial computarizada (TAC) del abdomen con cortes finos, simple y contrastada (Figura 1), o en su defecto, una resonancia magnética (RM). La centellografía con somatostatina (CS) y el ultrasonido endoscópico (UE) (Figura 2) pueden ser apropiados en casos de duda diagnóstica, con una sensibilidad del 82 al 90\% para este último método de imagen. ${ }^{11}$

En pacientes con lesiones recurrentes o cuando otros métodos de localización han sido equívocos, se puede realizar una inyección selectiva de calcio en las arterias pancreáticas, y posteriormente, medir los niveles de insulina en la vena hepática derecha o izquierda (procedimiento de Imamura-Doppman). ${ }^{12}$

A raíz del consenso de la ENETS (European Neuroendocrine Tumor Society), ${ }^{13}$ se sugiere que ante hallazgos por imagen sospechosos de un TNE en pacientes asintomáticos, se realice un test de sinaptofisina, polipéptido pancreático y cromogranina A. ${ }^{2}$ Esta última se ha utilizado como un marcador diagnóstico y pronóstico, ya que se eleva en un $60 \%$ de los casos de insulinomas; ${ }^{14,15}$ cifras mayores al doble de lo normal se asocian a una pobre supervivencia ${ }^{16}$ y mala respuesta al manejo con everolimus. ${ }^{17}$

En todos los pacientes se debe realizar un interrogatorio personal y familiar intencionado

Cuadro VI. Clasificación de los TNE según la OMS 2010.

\begin{tabular}{|c|c|c|c|c|}
\hline & \multicolumn{2}{|c|}{ Tumor neuroendocrino bien diferenciado } & \multirow{2}{*}{$\begin{array}{c}\text { Carcinoma } \\
\text { neuroendocrino } \\
\text { bien diferenciado }\end{array}$} & \multirow{2}{*}{$\begin{array}{c}\text { Carcinoma } \\
\text { neuroendocrino } \\
\text { mal diferenciado }\end{array}$} \\
\hline & $\begin{array}{c}\text { Comportamiento } \\
\text { benigno }\end{array}$ & $\begin{array}{c}\text { Comportamiento } \\
\text { incierto }\end{array}$ & & \\
\hline Tumor & $<2 \mathrm{~cm}$ & $>2 \mathrm{~cm}$ & Invasión local & Células pequeñas \\
\hline Mitosis & $<2$ mitosis $*$ & $>2$ mitosis & $>10$ mitosis & $>20$ mitosis \\
\hline Ki-67 & $<2 \%$ & $>2 \%$ & $>5 \%$ & $>15 \%$ \\
\hline $\begin{array}{l}\text { Invasión } \\
\text { vascular }\end{array}$ & $\begin{array}{l}\text { Sin invasión } \\
\text { vascular }\end{array}$ & Invasión vascular & $\begin{array}{c}\text { Invasión vascular } \\
\text { y metástasis }\end{array}$ & $\begin{array}{c}\text { Invasión vascular } \\
\text { y perineural }\end{array}$ \\
\hline
\end{tabular}

* Número de mitosis en 10 campos de alto poder. Ki-67 = índice de proliferación celular. 
para búsqueda de neoplasia endocrina múltiple (NEM) 1,18 por un $5 \%$ de asociación a este síndrome.

\section{Tratamiento}

Con independencia del grado histológico y potencial maligno, prácticamente todos los insulinomas deben ser resecados debido a las complicaciones metabólicas que suelen acompañarlos. ${ }^{2}$

Con base en el hecho de que, por lo general, tienen un comportamiento benigno, el tratamiento de elección para los tumores primarios de localización periférica sin sospecha de malignidad y menores a dos centímetros es la enucleación, siempre y cuando se encuentren a una distancia prudente del conducto pancreático principal (Figura 3). ${ }^{2}$ Este procedimiento puede ser realizado por laparoscopia o por cirugía abierta para tumores únicos y localizados. Si la enucleación no es posible, se deberá llevar a cabo una resección quirúrgica, la cual consistirá en una duodenopancreatectomía cefálica (procedimiento de Whipple) para tumores localizados en la cabeza o cuello del páncreas, o bien, una pancreatectomía distal para tumores de cuerpo y cola (Figura 4).

Cuando se sospeche de una lesión benigna, se deberá procurar conservar el bazo; en cambio, si la lesión sugiere malignidad o el tumor compromete los vasos esplénicos, se habrá de realizar una esplenectomía.

En los casos de "incidentalomas" (tumores no funcionales diagnosticados incidentalmente por estudios de imagen), se puede optar por un manejo conservador y vigilancia en casos seleccionados, ${ }^{19}$ teniendo como criterio un volumen tumoral en un rango de entre uno y tres centímetros, según diferentes autores; ${ }^{20}$ ante esta ambigüedad, las guías del $\mathrm{NCCN}^{2}$ (National Comprehensive Cancer Network) sugieren, con un bajo nivel de evidencia, que el tamaño máximo debe ser de un centímetro. Ante tumores entre uno y dos centímetros, recomiendan ya sea la enucleación o la resección quirúrgica asegurando márgenes negativos, y ante tumores de dos centímetros o mayores, realizar, además una linfadenectomía por un pequeño pero real riesgo de metástasis. $^{2}$

En caso de enfermedad metastásica limitada al hígado, una resección quirúrgica del tumor primario y una metastasectomía pueden ser consideradas con intenciones curativas en pacientes seleccionados, ya sea en cirugía de uno o dos tiempos, ${ }^{2}$ con una supervivencia reportada en un rango entre 41 y $100 \% .{ }^{21}$ Una cirugía citorreductora no curativa puede, además, ser contemplada para algunos pacientes a fin de reducir la producción de hormonas y paliar los síntomas. ${ }^{2}$
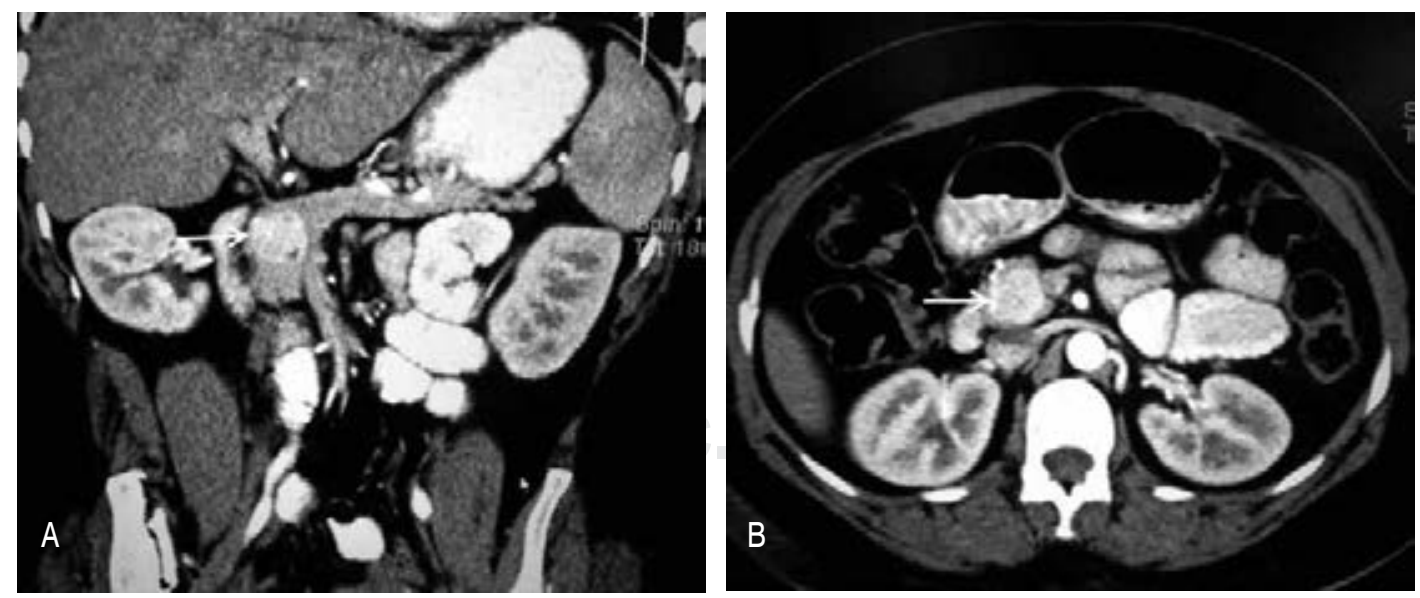

Figura 1. A y B. Tomografía axial contrastada en la cual se observa una tumoración en la cabeza del páncreas con dimensiones de $2.6 \times 2.4 \mathrm{~cm}$, de morfología redondeada y bordes regulares, interfaces conservadas y con realce heterogéneo tras la administración del medio de contraste. 


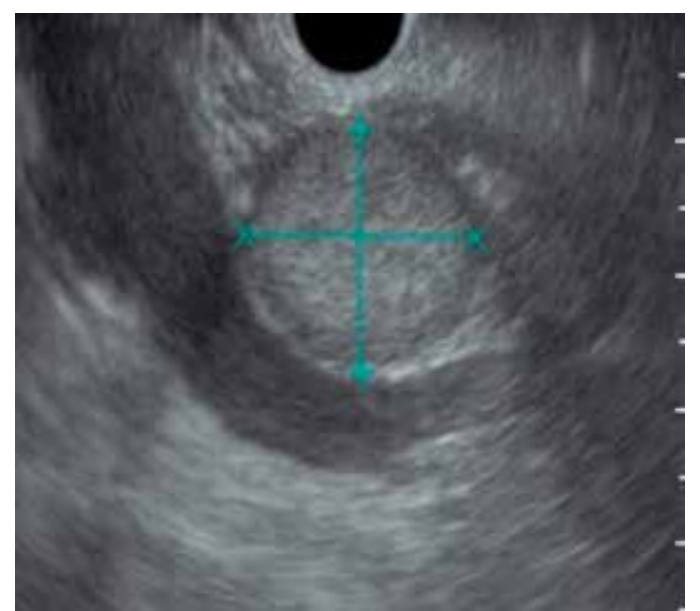

Figura 2. Ultrasonido endoscópico pancreático en el cual se observa un tumor correspondiente a insulinoma, con dimensiones de $2.2 \times 2.1 \mathrm{~cm}$, con relación estrecha hacia la vena porta.

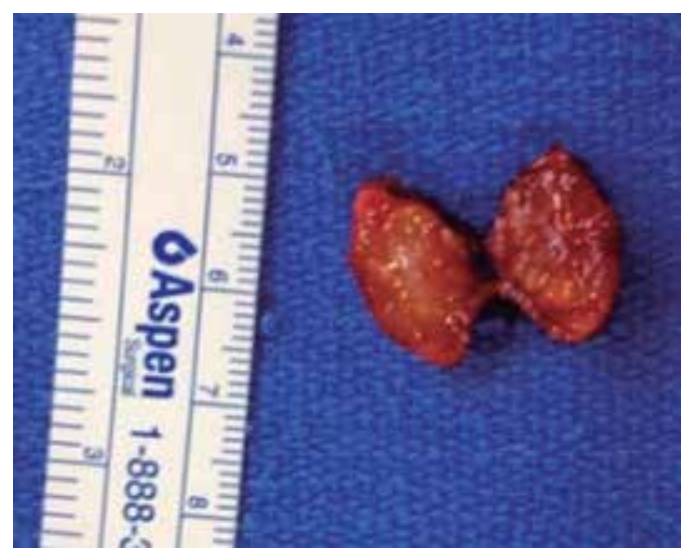

Figura 3. Pieza quirúrgica posterior a la enucleación por insulinoma, de color pardo rojizo y con bordes bien delimitados.

El trasplante hepático se ha realizado en pacientes con metástasis no resecables y confinadas al hígado, ${ }^{22}$ aunque la mayoría de ellos finalmente desarrolló recurrencias; ${ }^{23}$ la evidencia actual no permite recomendarlo como una conducta estandarizada. ${ }^{2}$

Para los casos de tumores no candidatos a resección, se podrá considerar el uso de terapia sistémica, para la cual se dispone de análogos de la somatostatina (octreotide de acción prolongada y lanreotide), quimioterapia

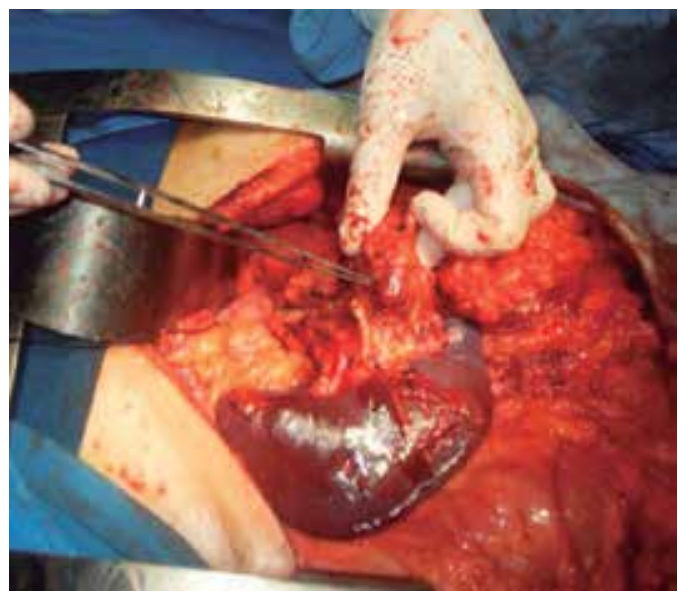

Figura 4. Pancreatectomía distal con esplenectomía; lesión tumoral en la cola del páncreas.

(5 fluorouracilo, capecitabine, dacarbazine, oxaliplatino, estreptozocina y temozolomide), terapia blanco (everolimus y sunitinib), interferones, entre otras opciones. ${ }^{2}$

\section{Seguimiento}

La duración óptima de la vigilancia es desconocida, pero se sabe que la mayoría de las recurrencias se aprecian en los primeros dos años, y todas las que se han reportado han sido en un periodo máximo de 10 años. ${ }^{24}$ Tampoco se ha logrado determinar el intervalo ideal entre revisiones; se sugieren rangos de entre tres y 12 meses en función del estado del paciente y del estatus - benigno o maligno- de la enfermedad. ${ }^{2}$ El seguimiento requiere de marcadores séricos en todos los casos y eventuales estudios de imagen (TAC o RM).

\section{CONCLUSIONES}

Los insulinomas son los tumores neuroendocrinos del páncreas más frecuentes y los que se asocian a un mejor pronóstico. La expresión clínica de estas lesiones es resultado de la sobreproducción de insulina, la cual se traduce en hipoglucemia. Un diámetro mayor a dos centímetros y un alto índice de mitosis son los mayores indicadores de enfermedad maligna. La mayoría de los insulinomas deberán ser tratados con intención curativa, incluso en lesiones suge- 
rentemente benignas; esto por los desórdenes hormonales que con frecuencia acompañan a estos tumores. La enucleación deberá ser la primera opción a considerar, aunque no todos los pacientes son candidatos a ella, seguida de resecciones quirúrgicas, cuya extensión estará en función de la localización del tumor. Otras opciones de manejo incluyen cirugías citorreductoras, metastasectomías, uso de quimioterapia, terapia blanco y análogos de la somatostatina.

\section{REFERENCIAS}

1. Medrano-Guzmán R. Tumores neuroendocrinos gastroenteropancreáticos. GAMO. 2011; 10 Supl 2: 17-24.

2. Neuroendocrine Tumors. NCCN Clinical Practice Guidelines in Oncology. Versión 2. 2016. Disponible en: https://www.nccn.org/professionals/physician_gls/ pdf/neuroendocrine.pdf

3. Halfdanarson TR, Rabe KG, Rubin J, Petersen GM. Pancreatic neuroendocrine tumors (PNETs): incidence, prognosis and recent trend toward improved survival. Ann Oncol. 2008; 19: 1727-1733.

4. Yao JC, Eisner MP, Leary C, Dagohoy C, Phan A, Rashid A, et al. Population-based study of islet cell carcinoma. Ann Surg Oncol. 2007; 14: 3492-3500.

5. Moore FD, Scoinski MA, Joste NE. Endocrine tumors and malignancies. In: Skarin A, editor. Atlas of diagnostic oncology. 3rd ed. Philadelphia: Elsevier Science Limited; 2003.

6. Modlin IM, Kidd M, Pfragner R, Eick GN, Champaneria MC. The functional characterization of normal and neoplastic human enterochromaffin cells. J Clin Endocrinol Metab. 2006; 91: 2340-2348.

7. Klimstra DS, Arnold R, Capella C, Hruban RH, Kloppel $\mathrm{G}$, Komminoth P, et al. Neuroendocrine neoplasms of the pancreas. In: Bosman FT, Carneiro F, Hruban RH, Theise N, eds. WHO classification of tumors of the digestive system. Lyon: WHO Press; 2010.

8. Vargas-Martínez CC, Castaño-Llano R. Tumores neuroendocrinos gastroenteropancreáticos. Rev Col Gastroenterol. 2010; 25: 165-176.

9. Zúñiga-Monge D. Tumores neuroendocrinos gastrointestinales. Med Leg Costa Rica. 2013; 30: 89-98.

10. Cryer PE, Axelrod L, Grossman AB, Heller SR, Montori VM, Seaquist ER, et al. Evaluation and management of adult hypoglycemic disorders: an Endocrine Society Clinical Practice Guideline. J Clin Endocrinol Metab. 2009; 94: 709-728.

11. James PD, Tsolakis AV, Zhang M, Belletrutti PJ, Mohamed R, Roberts DJ, et al. Incremental benefit of preoperative EUS for the detection of pancreatic neuroendocrine tumors: a meta-analysis. Gastrointest Endosc. 2015; 81: 848-856.

12. Doppman JL, Chang R, Fraker DL, Norton JA, Alexander $H R$, Miller DL, et al. Localization of insulinomas to regions of the pancreas by intra-arterial stimulation with calcium. Ann Intern Med. 1995; 123: 269-273.

13. Klöppel G, Couvelard A, Perren A, Komminoth P, McNicol AM, Nilsson O, et al. ENETS Consensus Guidelines for the Standards of Care in Neuroendocrine
Tumors: towards a standardized approach to the diagnosis of gastroenteropancreatic neuroendocrine tumors and their prognostic stratification. Neuroendocrinology. 2009; 90: 162-166.

14. Bernini GP, Moretti A, Ferdeghini M, Ricci S, Letizia $\mathrm{C}, \mathrm{D}^{\prime}$ Erasmo $\mathrm{E}$, et al. A new human chromogranin ' $A$ ' immunoradiometric assay for the diagnosis of neuroendocrine tumours. Br J Cancer. 2001; 84: 636-642.

15. Campana D, Nori F, Piscitelli L, Morselli-Labate AM, Pezzilli R, Corinaldesi R, et al. Chromogranin A: is it a useful marker of neuroendocrine tumors? J Clin Oncol. 2007; 25: 1967-1973.

16. Ter-Minassian M, Chan JA, Hooshmand SM, Brais LK, Daskalova A, Heafield R, et al. Clinical presentation, recurrence, and survival in patients with neuroendocrine tumors: results from a prospective institutional database. Endocr Relat Cancer. 2013; 20: 187-196.

17. Yao JC, Pavel M, Phan AT, Kulke MH, Hoosen S, St Peter J, et al. Chromogranin A and neuron-specific enolase as prognostic markers in patients with advanced pNET treated with everolimus. J Clin Endocrinol Metab. 2011; 96: 3741-3749.

18. Alexakis N, Neoptolemos JP. Pancreatic neuroendocrine tumours. Best Pract Res Clin Gastroenterol. 2008; 22: 183-205.

19. Lee LC, Grant CS, Salomao DR, Fletcher JG, Takahashi $\mathrm{N}$, Fidler JL, et al. Small, nonfunctioning, asymptomatic pancreatic neuroendocrine tumors (PNETs): role for nonoperative management. Surgery. 2012; 152: 965-974.

20. Regenet N, Carrere N, Boulanger G, de Calan L, Humeau $\mathrm{M}$, Arnault V, et al. Is the 2-cm size cutoff relevant for small nonfunctioning pancreatic neuroendocrine tumors: A French multicenter study. Surgery. 2016; 159: 901-907.

21. Lesurtel M, Nagorney DM, Mazzaferro V, Jensen RT, Poston $\mathrm{GJ}$. When should a liver resection be performed in patients with liver metastases from neuroendocrine tumours? A systematic review with practice recommendations. HPB (Oxford). 2015; 17: 17-22.

22. Bonaccorsi-Riani E, Apestegui C, Jouret-Mourin A, Sempoux C, Goffette P, Ciccarelli O, et al. Liver transplantation and neuroendocrine tumors: lessons from a single centre experience and from the literature review. Transpl Int. 2010; 23: 668-678.

23. Basturk O, Yang Z, Tang LH, Hruban RH, Adsay V, McCall CM, et al. The high-grade (WHO G3) pancreatic neuroendocrine tumor category is morphologically and biologically heterogenous and includes both well differentiated and poorly differentiated neoplasms. Am J Surg Pathol. 2015; 39: 683-690.

24. Strosberg JR, Cheema A, Weber JM, Ghayouri M, Han G, Hodul PJ, et al. Relapse-free survival in patients with nonmetastatic, surgically resected pancreatic neuroendocrine tumors: an analysis of the AJCC and ENETS staging classifications. Ann Surg. 2012; 256: 321-325.

Correspondencia:

Gustavo Alain Flores-Rangel

Hospital General de México

"Dr. Eduardo Liceaga"

Dr. Balmis No.148, Col. Doctores,

Delegación Cuauhtémoc, CDMX,

C.P. 06726, Teléfono: 01(55) 27892000.

Tel. particular: 5544478005 .

E-mail: clinicadepancreas@gmail.com 